\title{
Recent progress in MHD simulations of the solar-terrestrial connection
}

\author{
FENG XueShang * \& ZUO PingBing \\ SIGMA Weather Group, State Key Laboratory of Space Weather, National Space Science Center, \\ Chinese Academy of Sciences, Beijing 100190, China
}

In recent years, considerable efforts on Magnetohydrodynamic (MHD) simulation have been made to better understand the solar-terrestrial connection, i.e. the propagation and evolution of solar wind and solar storm as well as their effects on terrestrial system. This paper briefly reviews these works based on the selected publications during 20132014 that cover the solar and solar wind model, solar windMoon interaction model, solar wind-magnetosphere coupling model, and magnetospheric model, etc.

It is one of the key elements of space weather prediction to gain the knowledge of the global background solar wind, which seriously relies on the results of heliospheric numerical models. Gressl et al. [1] made a comparative study of MHD modeling of the solar wind by testing the feasibility and performance of three models: MAS/ENLIL (MHD Algorithm outside a Sphere/ENLIL), WSA/ENLIL (WangSheeley-Arge/ENLIL), and MAS/MAS. The model results are respectively compared with in-situ measurements of ACE and WIND near 1 AU. All the three models are able to well reproduce the large-scale structure of the background solar wind, but have certain uncertainties in predicting the arrival times of high-speed solar wind streams. It reveals by comparison of model running with synoptic magnetic maps from different observatories that, the model performance is significantly affected by the choice of the synoptic map.

Oran et al. [2] developed a three-dimensional global Alfvén Wave Solar Model within the extended-MHD approximation. It has the ability of simulating the heliospheric environment from upper chromosphere out to 1-2 AU. The exchange of energy and momentum between the plasma and Alfvénic wave field is described by the self-consistent coupling of two-temperature MHD equations and a wave kinetic equation. Compared with previous global models, it

*Corresponding author (email: fengx@ spaceweather.ac.cn) employs a unified treatment of calculating the wave dissipation in both open and closed magnetic field lines. The simulation efforts for modeling Carrington rotation 2063 are in great agreement with both the observations of thermal structure of the lower corona from SOHO, Hinode, and STEREO, and in-situ measurements of the solar wind structure beyond the Earth's orbit.

Computing methods of graphic processing unit (GPU) have been proven to be very valuable in various areas of scientific research due to their strong computational power. A novel application of GPU to the space weather modeling has recently been presented by Feng et al. [3]. For the purpose of computational speedup, GPU programming is realized for the SIP-CESE (Solar Interplanetary-Conservation Element and Solution Element) MHD model that investigates the solar corona and solar wind in interplanetary space. Their initial tests with available hardware show speedups of roughly $5 \mathrm{X}$ compared to traditional software implementation, pointing to the promising of GPU-accelerated-computing for space weather prediction with lead time. Zhou and Feng [4] proposed an improved space-time CESE numerical method to solve the MHD equations by applying a non-staggered space-time mesh system and improving the calculation of flow variables. It is demonstrated that the improved CESE scheme can enhance the accuracy of the solution when a globally fixed time step is used. When Courant-Friedrichs-Lewy (CFL) number is less than 0.1 , the scheme can significantly reduce the numerical dissipation but retain the solution quality. Using the simulation of the SIP-CESE model, Zhou et al. [5] studied the evolution of one large coronal mass ejection in the corona and heliosphere. It is confirmed by comparing the simulation results with measurements of STEREO and WIND that, both the fine solar coronal structure and the typical large-scale structure of the shock propagation can be successfully re- 
produced by the MHD simulation. Additionally, the shock arrival time at the Earth is estimated by the model with an error of $\sim 2 \mathrm{~h}$.

A 3-D global MHD model of the interaction between solar wind and the Moon was presented by Xie et al. [6]. Simulations with different IMF conditions reveal that basic characteristics of the lunar wake including the increase of central wake magnetic field and the decrease of plasma density can always be formed. It also shows that an acceleration region may appear and the plasma temperature is enhanced in the near wake. It is verified that fast magnetosonic mode rarefaction waves propagate away from the limb when the plasma moves into the lunar wake. The simulation results are well consistent with the observations from the WIND spacecraft.

Global 3-D MHD simulations are one of the most effective approaches to investigate the coupling between the solar wind and the magnetosphere. Wang et al. [7] introduced a global MHD model named the PPMLR-MHD model developed by their research group that has the advantage of low numerical dissipation and high order spatial accuracy. The framework of this model and recent advances in simulating the Solar-Magnetosphere-Ionosphere coupling system using the PPMLR-MHD model is briefly reviewed in that paper. Recently, Zhang et al. [8] have introduced an improved method to calculate the equivalent current systems (ECSs) using the global PPMLR-MHD model. The geomagnetic perturbation as a function of the interplanetary magnetic field (IMF) clock angle calculated from the ECS derived from both methods is compared with the observations. It indicates that the improved method can improve the simulation performance. It is also found that the incomplete counterbalance of the geomagnetic effect produced by the field-aligned current (FAC) and the ionospheric poloidal current contributes to most of the eastward-westward component of geomagnetic perturbation. Based on the PPMLRMHD model, Han et al. [9] investigated the energy budget of the magnetosphere during the well-known super geomagnetic storm occurring on November 20, 2003. The total energy input of this super storm is estimated in this way to be more than ten times of a moderate storm. The ratio of the injection of ring current to the total energy output is found to increase when the magnetospheric activity is enhanced.
Recently, the global MHD magnetosphric model BATSR-US has been improved by Meng et al. [10]. In the extended model, the pressure anisotropy is taken into account. The anisotropic MHD model is coupled with two inner magnetospheric models: CRCM Model (Comprehensive Ring Current Model) and RCM Model (Rice Convection Model). The simulations of the coupled global magnetosphere models indicated that pressure anisotropy is significant in controlling the configuration of magnetospheirc magnetic field, and it leads to a smaller earthward plasma jet from the tail reconnection site, a shorter magnetotail and a thinner magnetosheath. The comparisons between the simulation results of the coupled global anisotropic MHD model and in-situ measurements for special event show that, the model is greatly improved in reproducing the inner magnetospheric flow speed and magnetic field in the magnetotail when the pressure anisotropy is included.

1 Gressl C, Veronig A M, Temmer M, et al. Comparative study of MHD modeling of the background solar wind. Solar Phys, 2014, 289, 1783

2 Oran R, van der Holst B, Landi E, et al. A global wave-driven magnetohydrodynamic solar model with a unified treatment of open and closed magnetic field topologies. Astrophys J, 2013, 778, 176

3 Feng X S, Zhong D K, Xiang C Q, et al. GPU-accelerated computing of three-dimensional solar wind background, Sci China Earth Sci, 2013, 56, 11: 1864-1880

4 Zhou Y F, Feng X S. An improved CESE method and its application to steady-state coronal structure simulation. Sci China Earth Sci, 2014, 57: 153-166

5 Zhou Y F, Feng X S, Zhao X H. Using a 3-D MHD simulation to interpret propagation and evolution of a coronalmass ejection observed by multiple spacecraft: The 3 April 2010 event. J Geophys Res Space Phys, 2014, 119: 9321-9333

6 Xie L H, Li L, Zhang Y T, et al. Three-dimensional MHD simulation of the lunar wake. Sci China Earth Sci, 2013, 56: 330-338

7 Wang C, Guo X C, Peng Z, et al. Magnetohydrodynamics (MHD) numerical simulations on the interaction of the solar wind with the magnetosphere: A review. Sci China Earth Sci, 2013, 56: 1141-1157

8 Zhang J J, Wang C, Tang B B. Improved method to derive equivalent current systems from global MHD simulations. Sci China Earth Sci, 2014, 57: 167-173

9 Han J P, Wang C, Li H. Energetics characteristics of the super magnetic storm on November 20, 2003 based on 3D global MHD simulation. Sci China Earth Sci, 2014, 57: 3035-3046

10 Meng X, Tóth G, Glocer A, et al. Pressure anisotropy in global magnetospheric simulations: Coupling with ring current models. J Geophys Res Space Phys, 2013, 118: 5639-5658 